\title{
Improving Damping of Power Networks: Power Scheduling and Impedance Adaptation
}

\author{
Enrique Mallada, Student Member, IEEE, and Ao Tang, Senior Member, IEEE \\ School of ECE, Cornell University, Ithaca, NY 14853
}

\begin{abstract}
This paper studies the effect of power scheduling and line impedances on the damping of a power network. We relate the damping of a network with the algebraic connectivity of a state dependent Laplacian. Via implicit function theorem, we further characterize its dependence on network parameters. This allows us to derive several updating directions that can locally improve the damping. The analysis also provides some interesting insight. For example, improving connectivity, by adding lines for instance, may not be beneficial in terms of damping.
\end{abstract}

\section{INTRODUCTION}

The stability of electrical power networks is one of the major concerns of every utility company. When a blackout occurs, the economic impact can be valued from hundreds of millions to several billion dollars [11]. Therefore, it is important to make sure that the operating point of the network is as good as possible. One suitable measure of what "good" means is the value of the rate of convergence, i.e. the damping, of the network.

Several models with different levels of details have been proposed to study the dynamical behaviors of power networks. In general, these models are classified into two main categories: network reduction models and network preserving models; see [3] for a fairly complete review of them. As the names suggest, only the later preserves the real topology of the network and can be used to capture several network dependent behaviors of the system.

In this paper we study the effect of network topology and parameters on the damping, i.e. the dominant eigenvalue, of the network preserving model introduced in [1]. We first relate the damping with the algebraic connectivity of a state dependent weighted Laplacian [2]. This evidences the interplay between damping and network topology. Then, we use implicit function theorem [12] to explore the dependence of the algebraic connectivity on network parameters. More specifically, we derive how power scheduling and line impedances affect the operating point of the network and predict the net effect of these changes on the algebraic connectivity. With these results, we provide updating rules that can improve the damping of a power network. The analysis further provides us some insights. For example, improving connectivity, by adding lines for instance, may not improve the damping.

The rest of the paper is organized as follows. Section II describes the network preserving model introduced in [1], summarizing its assumptions. Section III relates the damping of a power network with the second smallest eigenvalue of a state dependent weighted Laplacian. We then characterize the dependence of the eigenvalue, a.k.a. algebraic connectivity, of this Laplacian in term of its weights in Section IV, and derive updating directions that improve the damping of a network in IV-A and IV-B. Numerical examples are presented in Section V and we conclude in Section VI.

\section{Network Preserving Model}

For the Bergen-Hill (BH) model introduced in [1], consider a power network composed of $n$ buses and $m$ generators, where each bus $i$ can potentially have attached a load, a generator, or both. The bus interconnection is modeled by lossless transmission lines connecting buses $i$ and $j$ with constant reactance $j x_{i j}$. Whenever $x_{i j}>0$ we say that the buses $i$ and $j$ are neighbors, and denote it by $i \in \mathcal{N}_{j}$ or $j \in \mathcal{N}_{i}$. See Figure 1(a) for an illustration of a sample power network with four buses and two generators.

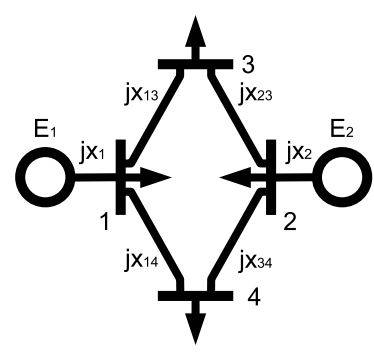

(a) Real Power Network

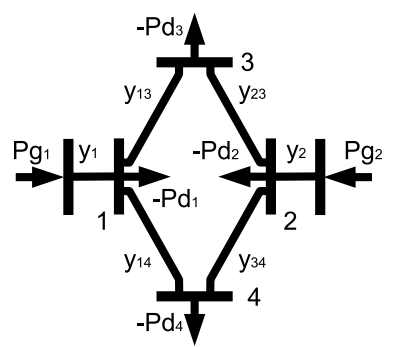

(b) Equivalent Network
Fig. 1. Power Network Representations

Each generator $i$ is assumed to have a constant internal voltage magnitude $\left|V_{i}\right|$ and transient reactance $j x_{i i}$. This assumption allows us to substitute the generator with a constant voltage internal bus and a lossless transmission line (with reactance $j x_{i i}$ ). Thus by transforming impedances in admittances (i.e. $y_{i j}=1 / j x_{i j}$ ) we obtain an equivalent representation of the network shown in Figure 1(b).

The state of each generator is completely described by the generator's phase $\phi_{i}$ and frequency deviation $\omega_{i}=\dot{\phi}_{i}$. The dynamics are modeled by the swing equation

$$
M_{i} \ddot{\phi}_{i}+D_{i} \dot{\phi}_{i}=P_{m_{i}}-P_{e_{i}}, \quad \forall i \in \mathcal{G}
$$

where $M_{i}$ and $D_{i}$ are the generator's inertia and damping, $P_{m_{i}}$ is the mechanical power, $P_{e_{i}}$ is the electrical real power that the network is demanding from the generator and $\mathcal{G}$ is the set of generator buses. 
The aggregated load of each bus $i$ is modeled by constant reactive power $Q_{d_{i}}$ and frequency dependent real power $P_{d_{i}}\left(\dot{\phi}_{i}\right)=P_{d_{i}}+D_{i} \dot{\phi}_{i}, \forall i \in \mathcal{L}$, with $\mathcal{L}$ being the set of load buses.

Then, under these assumptions, the dynamic behavior of [1] can be compactly described by

$$
M \ddot{\phi}+D \dot{\phi}=-B\left(f(b) \circ \sin \left(B^{T} \phi\right)\right)+P,
$$

where $(a \circ b)_{i}=a_{i} b_{i}$ is the Hadamard product between the vectors $a$ and $b$, and $(f(b))_{i j}=\left|V_{i}\right|\left|V_{j}\right| b_{i j}$ is the maximum instantaneous power flow between $i$ and $j .\left|V_{i}\right|$ is the voltage magnitude at bus $i$ and $b_{i j}$ is the imaginary part of the $i j^{\text {th }}$ element of the bus admittance matrix $Y_{b u s}$ [15]. The diagonal matrices $M$ and $D$ represent the generators' inertia, generators' damping and loads' frequency coefficients, i.e.

$$
(M)_{i j}=\left\{\begin{array}{l}
M_{i}, \text { if } i=j i \in \mathcal{G}, \\
0 \text { o.w., }
\end{array}(D)_{i j}=\left\{\begin{array}{l}
D_{i} \text { if } i=j, \\
0 \text { o.w. }
\end{array}\right.\right.
$$

we will use $D_{\max }, D_{\min }$ and $M_{\max }$ to denote the nonzero extreme values $D$ and $M$ can reach. The vector $P \in \mathbb{R}^{m+n}$ is the power injection at each bus, i.e.

$$
(P)_{i}=\left\{\begin{array}{cl}
P_{m_{i}} & \forall i \in \mathcal{G} \\
-P_{d_{i}} & \forall i \in \mathcal{L}
\end{array}\right.
$$

And the matrix $B \in \mathbb{R}^{(m+n) \times(m+n)(m+n-1)}$ is the incidence matrix of the complete graph $K_{m+n}=\left(\mathcal{V}, \mathcal{E}_{k}\right)$ defined for each bus $i \in \mathcal{V}=\mathcal{G} \cup \mathcal{L}$ and each edge $e \in \mathcal{E}_{k}$ as $B(i, e)$ equal 1 if $i$ is the head of $e,-1$ if $i$ is the tail of $e$, and 0 otherwise.

Note that although an orientation is implicit in the definition of $B$, equation (1) is independent of the orientation used. Also, by definition the range of $B$ is the $\operatorname{ker}\left(\mathbf{1}_{m+n}^{T}\right)$ where $\mathbf{1}_{m+n}$ is the vector of all ones of dimension $m+n$, i.e. $\mathbf{1}_{m+n}^{T} B=\mathbf{0}_{(m+n)(m+n-1)}^{T}$.

Remark 1: The matrix $B$ as defined here does not capture (alone) the topology of the network. It is the conjunction of $B$ and $b=\left(b_{i j}\right)$ that captures the topology since $b_{i j}>0$ if and only if $(i, j)$ represent a line of the extended power network graph $G=(\mathcal{V}, \mathcal{E})$, i.e. iff $i j \in \mathcal{E}$. In this way the addition of a line does not change the dimension of $B$.

\section{EFFECT OF TOPOLOGY}

The damping of (1) can be locally estimated by computing the eigenvalues of the Jacobian $J_{\phi^{*}}$ of the linearized version of (1) around a given equilibrium $\left(\phi^{*}, \dot{\phi}^{*}=0\right)$,

$$
M \delta \ddot{\phi}+D \delta \dot{\phi}+L\left(w\left(\phi^{*}\right)\right) \delta \phi=0,
$$

where the matrix $L\left(w\left(\phi^{*}\right)\right):=B \operatorname{diag}\left[w\left(\phi^{*}\right)\right] B^{T}$ represents the weighted Laplacian of the graph $G$ with weights $w_{i j}\left(\phi^{*}\right)=\left|V_{i}\right|\left|V_{j}\right| b_{i j} \cos \left(\phi_{j}^{*}-\phi_{i}^{*}\right)$, and captures several topological properties of the network (see e.g. [2]).

When $\left(\phi^{*}, 0\right)$ is stable, $L\left(w\left(\phi^{*}\right)\right)$ is positive semidefinite with, under generic conditions, only one zero eigenvalue $\nu_{1}\left(L\left(w\left(\phi^{*}\right)\right)\right)$ with eigenvector $\mathbf{1}_{m+n}$. This implies that the smallest eigenvalue of (2), $\lambda_{1}$, is zero. The existence of this zero eigenvalue is due to the rotational symmetry of the system, $\sin \left(B^{T}\left(\phi+\mathbf{1}_{m+n}\right)\right)=\sin \left(B^{T} \phi\right)$.
If $D_{i} \gg M_{i}$, it is possible to approximate (1) by setting $M=0$ [5]. Then, (2) becomes a first order system and the damping can be upper-bounded by,

$$
\Re\left[\lambda_{2}\right] \leq-\cos \left(\alpha^{*}\right) \frac{\nu_{2}\left(L\left(w\left(\phi^{*}\right)\right)\right)}{D_{\max }},
$$

where $\alpha^{*}:=\angle(D \mathbf{1}, \mathbf{1})$ is the angle between vectors $D \mathbf{1}$ and 1 ; we use $\Re[\cdot]$ and $\Im[\cdot]$ to denote the real and imaginary part of a complex element.

Equation (3) suggests a correlation between $\Re\left[\lambda_{2}\right]$ and the algebraic connectivity $\nu_{2}\left(L\left(w\left(\phi^{*}\right)\right)\right)$; however, a priori this relation seems to be only valid when $D_{i} \gg M_{i}$. The problem is that when $D_{i} \ngtr M_{i}$, the computation of the eigenvalues of (2) is usually done by introducing the state variables $\delta \omega=\delta \dot{\phi}$ and interpreting (2) as a first order linear system of dimension $2 m+n$. This approach hides the rich symmetry inherent to $M, D$ and $L\left(w\left(\phi^{*}\right)\right)$ and makes the generalization of (3) hard.

In this paper we use a more elegant approach to relate the damping of (1) with $\nu_{2}\left(L\left(w\left(\phi^{*}\right)\right)\right)$. Using Matrix Polynomial Theory [8], we show that the when the network is close to a bifurcation [4] (3) still holds. This approach is summarized next.

Instead of solving the linear eigenvalue problem of finding pairs $\left(\lambda_{i}, v_{i}\right) \in \mathbb{C} \times \mathbb{C}^{2 m+n}$ such that $\left(\lambda_{i} I-J_{\phi^{*}}\right) v_{i}=0$, we solve the quadratic eigenvalue problem [16] of finding $\left(\lambda_{i}, x_{i}\right) \in \mathbb{C} \times \mathbb{C}^{m+n}$ such that $Q\left(\lambda_{i}\right) x_{i}=0$ with $Q(\lambda)=$ $M \lambda^{2}+D \lambda+L\left(w\left(\phi^{*}\right)\right)$. It is easy to show that $Q(\lambda)$ has $2(m+n)$ eigenvalues and that if $\lambda_{i}$ is an eigenvalue of $J_{\phi^{*}}$, it is also an eigenvalue of $Q(\lambda)$ [8]. The difference in the number of eigenvalues is due to the fact that $M$ has $n$ zero eigenvalues, which is reflected in $Q(\lambda)$ with the presence of $n$ infinite eigenvalues. However, since we are only concerned about the dominant eigenvalue of (2), these infinite eigenvalues are not of interest to us.

This is a more natural formulation, since now the symmetry of $M, D$ and $L\left(w\left(\phi^{*}\right)\right)$ implies that if $x_{i}$ is a right eigenvector of $Q(\lambda)$ then its complex conjugate $\bar{x}_{i}$ is a left eigenvector, and given the finite pair $\left(\lambda_{i}, x_{i}\right)$ the following relationship holds

$$
\lambda_{i}= \begin{cases}-\frac{l\left(x_{i}\right)}{d\left(x_{i}\right)} & \text { if } m\left(x_{i}\right)=0, \\ \frac{-d\left(x_{i}\right) \pm \sqrt{d\left(x_{i}\right)^{2}-4 m\left(x_{i}\right) l\left(x_{i}\right)}}{2 m\left(x_{i}\right)} & \text { otherwise. }\end{cases}
$$

where $m(x)=\bar{x}^{T} M x, d(x)=\bar{x}^{T} D x$ and $l(x)=$ $\bar{x}^{T} L\left(w\left(\phi^{*}\right)\right) x$. Notice that since $M \geq 0, D>0$ and $L\left(w\left(\phi^{*}\right)\right) \geq 0, m(x), d(x)$ and $l(x)$ are real and for any $x \neq 0, m(x) \geq 0, d(x)>0$ and $l(x) \geq 0$.

The next theorem extends (3) to some cases where $M \neq 0$.

Theorem 1 (Damping Bound): When (1) is close to a bifurcation, the dominant eigenvalue $\lambda_{2}$ of (2) is real and bounded by (3).

Proof: Since the system is assumed to be close to a bifurcation, then $\Re\left[\lambda_{2}\right]$ must close to the imaginary axis, i.e. $\left|R e\left[\lambda_{2}\right]\right| \ll \frac{D_{\min }}{M_{\max }}$. Thus, it follows from (4) that $\lambda_{2}$ is real $\left(\Im\left[\lambda_{2}\right]=0\right)$. 
We first show that $\Re\left[\lambda_{2}\right] \leq-\frac{l\left(x_{2}\right)}{d\left(x_{2}\right)}$, which is trivial from (4) if $m\left(x_{2}\right)=0$. Thus consider the case of $m\left(x_{2}\right)>0$. Since $\Im\left[\lambda_{2}\right]=0$, (4) implies $d\left(x_{2}\right)^{2}>4 m\left(x_{2}\right) l\left(x_{2}\right)$. Thus, using the fact that $\sqrt{1-x} \leq 1-\frac{1}{2} x$ and (4), we get

$\Re\left[\lambda_{2}\right] \leq \frac{-d\left(x_{2}\right)+d\left(x_{2}\right)\left(1-\frac{1}{2}\left(\frac{4 m\left(x_{2}\right) l\left(x_{2}\right)}{d\left(x_{2}\right)^{2}}\right)\right)}{2 m\left(x_{2}\right)}=-\frac{l\left(x_{2}\right)}{d\left(x_{2}\right)}$.

Therefore, whenever $\Im\left[\lambda_{2}\right]=0, \Re\left[\lambda_{2}\right]=\lambda_{2} \leq-\frac{l\left(x_{2}\right)}{d\left(x_{2}\right)}$.

The main problem with this bound is that since $l(x)$ is not positive definite, it cannot be readily lower bounded by a positive value. We therefore need to use the fact that $x_{2}$ is an eigenvector of $Q(\lambda)$ to obtain an appropriate lower bound on $l\left(x_{2}\right)$.

Since $x_{2}$ is an eigenvector and $\lambda_{2} \neq 0$, then it follows from $\mathbf{1}_{m+n}^{T} L_{\phi^{*}}=0$ and $Q\left(\lambda_{2}\right) x_{2}=0$ that,

$$
\begin{aligned}
0 & =\mathbf{1}_{m+n}^{T} Q\left(\lambda_{2}\right) x_{2}=\mathbf{1}_{m+n}^{T}\left(\lambda_{2}^{2} M+\lambda_{2} D+L_{\phi^{*}}\right) x_{2} \\
& =\mathbf{1}_{m+n}^{T}(\lambda M+D) x_{2} .
\end{aligned}
$$

So, when $\lambda_{2} \ll \frac{D_{\min }}{M_{\max }}, x_{2} \in \operatorname{ker}\left[\mathbf{1}_{m+n}^{T} D\right]$ and it follows that the biggest angle that $x_{2}$ can achieve with respect to $\operatorname{ker}\left(\mathbf{1}^{T}\right)$ is $\alpha^{*}$ and therefore $l\left(x_{2}\right) \geq \cos \left(\alpha^{*}\right)\left\|x_{2}\right\|^{2}>0$. Finally, since $d\left(x_{2}\right) \leq D_{\max }\left\|x_{2}\right\|^{2}$, we get (3).

\section{IMPROVING DAMPING OF A StABle EQUilibriUm}

This section introduces some updating rules on the network parameters that improve the damping of a power network close to a saddle node bifurcation. In order to do this we need to know how the second smallest eigenvalue $\nu_{2}(L(w))=: \nu_{2}(w)$ of the Laplacian $L(w)$ changes with $w$. There are several nice properties of $\nu_{2}(w)$ when $L(w)$ is positive semidefinite, see e.g. [2]. In particular, $\nu_{2}(w)$ is a concave function of $w$ and homogeneous of degree one, i.e. $\nu_{2}(\lambda w)=\lambda \nu_{2}(w)$.

Here we are interested in computing $\frac{\partial}{\partial w_{i j}} \nu_{2}(w)$ whenever it is possible. If for given $w$ the multiplicity of $\nu_{2}(w)$ is one, $\nabla_{w} \nu_{2}(w)$ is defined and can be readily computed by expressing $\nu_{2}(w)$ as

$$
\begin{aligned}
\nu_{2}(w) & =\min _{\{x:\|x\|=1,\langle x, \mathbf{1}\rangle=0\}} x^{T} L(w) x \\
& =\min _{x} \max _{\mu_{1}, \mu_{2}} W\left(w, x, \mu_{1}, \mu_{2}\right) \\
& =x^{*}(w)^{T} L(w) x^{*}(w)
\end{aligned}
$$

where $W\left(w, x, \mu_{1}, \mu_{2}\right)$ is the Lagrangian and $x^{*}(w)$ is the unique eigenvector corresponding to $\nu_{2}(w)$. Then, we can use envelope theorem [14] to compute

$$
\frac{\partial \nu_{2}(w)}{\partial w_{i j}}=\left(x^{*}(w)_{i}-x^{*}(w)_{j}\right)^{2}
$$

Thus, the gradient can be compactly expressed as $\nabla_{w} \nu_{2}(w)=p_{x^{*}(w)}$, where $p_{x^{*}(w)}:=$ $\operatorname{diag}\left[B^{T} x^{*}(w) x^{*}(w)^{T} B\right] \mathbf{1}_{m+n}$, and $\operatorname{diag}[A]$ is the matrix operator that projects all the off diagonal elements to zero and keeps the diagonal untouched. Similarly, we will use $\operatorname{diag}[a]$ to denote the operator that converts the vector $a$ in a diagonal matrix.

When $\nu_{2}(w)$ is not simple, there are several $x^{*}(w)$ that solve this optimization problem. In this case $\nabla_{w} \nu_{2}(w)$ is in general not defined, but it is easy to show that for every $x^{*}(w), p_{x^{*}(w)} \in \partial_{w}^{+} \nu_{2}(w)$, where $\partial^{+} g(w):=$ $\{p \mid\langle p, \bar{w}-w\rangle \geq g(\bar{w})-g(w)\}$ is the concave superdifferential of the function $g(w)$. Although in general there is no guarantee of local improvement for every direction $p \in$ $\partial^{+} g(w)$, subgradient-type iterations can still reach the global optimum. See [10] for general treatment of subdifferentials of eigenvalues of symmetric matrices.

One interesting consequence of this derivation is that $\left(p_{x^{*}(w)}\right)_{i j} \geq 0$. This implies that $\nu_{2}(w)$ is a nondecreasing function of its elements and the only way to reduce its value is by decreasing some $w_{i j}$. The main difficulty in our case is that the weights $w_{i j}$ depend on the parameters of the system in a nonlinear manner, i.e. $w_{i j}=\left|V_{i}\right|\left|V_{j}\right| b_{i j} \cos \left(\phi_{j}^{*}-\phi_{i}^{*}\right)$ where $\phi^{*}$ is a solution to

$$
F(\phi, b, P)=-B f(b) \circ \sin \left(B^{T} \phi\right)+P=0,
$$

for fixed line inductances $b$ and fixed power schedule $P$. Therefore, it is not clear at first sight how changes on $b$ and $P$ affect the corresponding $w$.

Throughout this paper we assume that the network is in a stable steady state such that the corresponding $\phi^{*}$ is stable and $L\left(w\left(\phi^{*}\right)\right)$ has only one zero eigenvalue, i.e. $\nu_{2}(w)>$ 0 . In the rest of this section we show how changing the network parameters affects $\nu_{2}(w)$, and how these changes are influenced by the topology of the network and the current operating point.

\section{A. Improving Damping via Power Scheduling}

In this subsection we show how power injection changes can locally improve the damping of a power network. We assume fixed line inductances $b=\left(b_{i j}\right)$ and full control of $P$ within the interior of feasible closed set $B_{P}=\left\{P: P_{\min } \leq\right.$ $\left.P \leq P_{\max }\right\}$. That is, we can not only change the values of $P_{g_{i}}$, but we can also change, up to a certain extent, $P_{d_{i}}$. Although this used to be an unreasonable assumption, the introduction of renewable energy sources in the distribution part of the network can enable the design of coordination mechanism that produce the desired changes on $P_{d}$.

Since $b$ is assumed to be fixed, $F(\phi, b, P)=F(\phi, P)$, and thus every equilibria $\phi^{*}$ satisfies, $F\left(\phi^{*}, P\right)=0$. Here, we will focus on how small changes in the power scheduling $P+\delta P$ affect the position of the equilibrium $\phi^{*}+\delta \phi$.

Although in principle $\delta \phi, \delta P \in \mathbb{R}^{m+n}$, only a subspace of $\mathbb{R}^{m+n}$ is of interest. Since the network is lossless, $\left\langle P, \mathbf{1}_{m+n}\right\rangle=0$ is always satisfied. Hence we will only consider changes $\delta P$ in the power schedule s.t. $\delta P \in$ $\operatorname{ker}\left(\mathbf{1}^{T}\right)$. Similarly, since $w\left(\phi^{*}+\kappa \mathbf{1}\right)=w\left(\phi^{*}\right) \forall \kappa \in \mathbb{R}$, we will restrict our attention on changes $\delta \phi \in \operatorname{ker}\left(\mathbf{1}^{T}\right)$. The relationship between $\delta P$ and $\delta \phi$ is then captured by

$$
F\left(\phi^{*}+\delta \phi, P+\delta P\right)=F(\delta \phi, \delta P)=0 .
$$


Theorem 2 (Controllability of $\delta \phi$ w.r.t $\delta P$ ): Given an equilibrium point $\phi^{*}$ of (1) with simple zero eigenvalue, and a power scheduling $P$ satisfying (5). There exists a neighborhood of $P, \mathcal{B}_{P} \subset P+\operatorname{ker}\left(\mathbf{1}^{T}\right)$, and function $\delta \phi(\delta P)$ such that $F(\delta \phi(\delta P), \delta P)=0, \quad \forall \delta P \in \mathcal{B}_{P}-P$ and $\delta \phi\left(\mathcal{B}_{P}\right)$ is open relatively to $\operatorname{ker}\left(\mathbf{1}_{m+n}^{T}\right)$, i.e. $\delta \phi$ is fully controllable by $\delta P$.

Moreover, $\frac{d(\delta \phi)}{d(\delta P)}$ can be computed as

$$
\frac{d(\delta \phi)}{d(\delta P)}=L\left(w\left(\phi^{*}\right)\right)^{\dagger}
$$

where $L\left(w\left(\phi^{*}\right)\right)^{\dagger}$ is the Moore-Penrose pseudoinverse of the weighted Laplacian $L\left(w\left(\phi^{*}\right)\right)$.

Proof: The proof of this theorem comes from applying implicit function theorem (see e.g. [12]) on a properly defined function. Notice that $\frac{\partial}{\partial \phi} F(\phi, P)=-L\left(w\left(\phi^{*}\right)\right)$, and $\frac{\partial}{\partial P} F(\phi, P)=I_{m+n}$. Thus, since $L\left(w\left(\phi^{*}\right)\right)$ is singular, implicit function theorem cannot be directly applied. However, our restriction of $(\delta \phi, \delta P)$ to the subspace $\operatorname{ker}\left(\mathbf{1}_{m+n}^{T}\right) \times \operatorname{ker}\left(\mathbf{1}_{m+n}^{T}\right)$ does not suffer this problem.

Since both vectors are restricted to $\operatorname{ker}\left(\mathbf{1}_{m+n}^{T}\right)$, by choosing orthonormal basis of column vectors $\left\{T_{j}\right\}$ we can write

$$
\delta \phi=T x \text { and } \delta P=T y
$$

where the matrix $T=\left[T_{j}\right] \in \mathbb{R}^{(m+n) \times(m+n-1)}$ is a full column rank matrix, $T^{T} T=I_{m+n-1}$ and $T T^{T}=$ $I_{m+n}-\frac{1}{m+n} \mathbf{1}_{m+n} \mathbf{1}_{m+n}^{T}$ is the orthogonal projection onto $\operatorname{ker}\left(\mathbf{1}_{m+n}^{T}\right)$.

Now, define $H(x, y)=T^{T} F(T x, T y)$. Since $\mathbf{1}_{m+n}^{T} B=$ $0, F(\phi, P) \in \operatorname{ker}\left(\mathbf{1}_{m+n}^{T}\right)$ provided $P \in \operatorname{ker}\left(\mathbf{1}_{m+n}^{T}\right)$. Thus, $F(T x, T y)=0$ if and only if $H(x, y)=0$, and $H$ represents the same constraints as $F$ when restricted to $\operatorname{ker}\left(\mathbf{1}^{T}\right) \times \operatorname{ker}\left(\mathbf{1}^{T}\right)$. Differentiating $H$ with respect $x$ and $y$ gives

$\frac{\partial}{\partial x} H(x, y)=-T^{T} L\left(w\left(\phi^{*}\right)\right) T$, and $\frac{\partial}{\partial y} H(x, y)=I_{m+n-1}$.

Since $\frac{\partial}{\partial x} H(x, y)$ is nonsingular, by implicit function theorem, there exist neighborhoods of $0, \mathcal{B}_{x}$ and $\mathcal{B}_{y}$, and a function $x(y)$ such that $H(x(y), y)=0$ and $x\left(\mathcal{B}_{y}\right)=\mathcal{B}_{x}$.

Finally, since $H(x(y), y)=0$ on $\mathcal{B}_{y}$,

$$
\begin{aligned}
\frac{d}{d x} H(x(y), y) & =\frac{\partial}{\partial x} H(x(y), P) \frac{d x(y)}{d y}+\frac{\partial}{\partial y} H(x(y), y) \\
& =-T^{T} L\left(w\left(\phi^{*}\right)\right) T \frac{d x(y)}{d y}+I_{m+n-1}=0
\end{aligned}
$$

and therefore $\frac{d x}{d y}=\left(T^{T} L\left(w\left(\phi^{*}\right)\right) T\right)^{-1}$.

Defining $\delta \phi(\delta P)=T x\left(T^{T} \delta P\right)$ and $\mathcal{B}_{P}=P+T \mathcal{B}_{y}$ gives the first statement of the theorem. Equation (7) follows from $\frac{d(\delta \phi)}{d(\delta P)}=T \frac{d x}{d y} T^{T},\left(T^{T} L\left(w\left(\phi^{*}\right)\right) T\right)^{-1}=T^{T} L\left(w\left(\phi^{*}\right)\right)^{\dagger} T$ and the fact that $T T^{T} L\left(w\left(\phi^{*}\right)\right)^{\dagger} T T^{T}=L\left(w\left(\phi^{*}\right)\right)^{\dagger}$.

Using (7) we can predict how small changes of the power affects the position of the equilibria, which in turn affects the value of $w(\phi)$ (recall $w_{i j}=\left|V_{i}\right|\left|V_{j}\right| b_{i j} \cos \left(\phi_{j}-\phi_{i}\right)$ ).
Thus, we can use Theorem 2 to compute the changes of the weights $\delta w$ as

$$
\begin{aligned}
\delta w & =\frac{d w}{d(\delta P)} \delta P=\left(\frac{\partial w}{\partial \phi}\right)\left(\frac{d(\delta \phi)}{d(\delta P)}\right) \delta P \\
& =\left(-\operatorname{diag}\left[f(b) \circ \sin \left(B^{T} \phi^{*}\right)\right] B^{T}\right)\left(L^{\dagger}\left(w\left(\phi^{*}\right)\right)\right) \delta P \\
& =: A\left(\phi^{*}\right) \delta P
\end{aligned}
$$

where we use the fact

$$
\frac{\partial w}{\partial \phi}=-\operatorname{diag}\left[f(b) \circ \sin \left(B^{T} \phi\right)\right] B^{T} .
$$

Ideally, we would like to move $\delta w \in \operatorname{span}\left(p_{x^{*}}\left(w\left(\phi^{*}\right)\right)\right)$, but we are constrained only to the subspace $\operatorname{span}\left(A\left(\phi^{*}\right)\right)$. Therefore, a natural alternative is to set $\delta P$ such that the corresponding $\delta w$ is the orthogonal projection of $p_{x^{*}(w)}$ onto $\operatorname{span}\left(A\left(\phi^{*}\right)\right)$. This is done by setting $\delta P=$ $\gamma A\left(\phi^{*}\right)^{\dagger} p_{x^{*}\left(w\left(\phi^{*}\right)\right)}$ which gives

$$
\delta w=A\left(\phi^{*}\right) \delta P=\gamma A\left(\phi^{*}\right) A\left(\phi^{*}\right)^{\dagger} p_{x^{*}\left(w\left(\phi^{*}\right)\right)} .
$$

Remark 2: Although the updating direction of this section modifies the values of $P_{g}:=\left(P_{g_{i}}\right)^{T}$ and $P_{d}:=\left(P_{d_{i}}\right)^{T}$, it is possible to constraint its actions only to $P_{g}$ by projecting $\delta P$ onto $S=\operatorname{ker}\left(\mathbf{1}_{m+n}^{T}\right) \cap B_{P}$ and setting $P_{d, \text { min }}=$ $P_{d, \max }=P_{d}$ in the definition of $B_{P}$. We denote this projection operation onto the set $S$ by $\Pi_{S}[\cdot]$, and similarly use $\Pi_{B_{P}}[\cdot]$ to define the analogous for $B_{P}$. Note that $\Pi_{B_{P}}[\cdot]$ enforces the constraint of $B_{P}$.

\section{B. Improving Damping via Impedance Adaption}

In this subsection we study how the changes of line inductances $b_{i j}$, due to changes in the network topology or the utilization of FACTS devices [9], can affect the operating point of a network. Using this result, we will show that it is possible that the addition of a line can weaken the condition of the network, i.e. $\nu_{2}(w)$ is reduced.

In order to measure how changes of $\delta b$ affect the weights $w$, we proceed in the same manner as in Theorem 2. We start by computing the total derivative $\frac{d w}{d(\delta b)}$ which is given by

$$
\frac{d w}{d(\delta b)}=\frac{\partial w}{\partial b}+\frac{\partial w}{\partial \phi} \frac{d(\delta \phi)}{d(\delta b)} .
$$

Since $w(\phi, b)=f(b) \circ \cos \left(B^{T} \phi\right)$, it is straightforward to show that

$$
\frac{\partial w}{\partial b}=\operatorname{diag}\left[v \circ \cos \left(B^{T} \phi\right)\right]
$$

where the elements of the vector $v$ are $(v)_{i j}=\left|V_{i}\right|\left|V_{j}\right|$ if $i j \in \mathcal{E}$ and $(v)_{i j}=0$ otherwise.

The main difficulty again rises in computing how the changes of $b$, i.e. $\delta b$, affect $\phi^{*}$. This is assessed in the next theorem. As in Theorem 2, we restrict our attention to $\delta \phi \in$ $\operatorname{ker}\left(\mathbf{1}^{T}\right)$. We do not impose any restriction on $\delta b$ besides the physical ones, i.e. $b+\delta b \in\left\{b: b_{\min } \leq b \leq b_{\max }\right\}$.

Theorem 3 (Controllability of $\delta \phi$ w.r.t $\delta b$ ): Given an equilibrium point $\phi^{*}$ of (1) with a simple zero eigenvalue, and bus admittances $b$ such that $F\left(\phi^{*}, b\right)=0$. There exists 
a neighborhood of $b, \mathcal{B}_{b}$, a neighborhood of $\phi^{*}, \mathcal{B}_{\phi^{*}}$, and function $\delta \phi(\delta b)$ such that

$$
F(\delta \phi(\delta b), \delta b)=0, \quad \forall \delta b \in \mathcal{B}_{b}-b
$$

and $\phi^{*}+\delta \phi\left(b+\mathcal{B}_{b}\right)=\mathcal{B}_{\phi^{*}}$. Moreover, $\frac{d(\delta \phi)}{d(\delta P)}$ can be computed as

$$
\frac{d(\delta \phi)}{d(\delta b)}=-L\left(w\left(\phi^{*}\right)\right)^{\dagger} B \operatorname{diag}\left[v \circ \sin \left(B^{T} \phi^{*}\right)\right] .
$$

Proof: Since $\delta \phi$ is restricted to $\operatorname{ker}\left(\mathbf{1}^{T}\right)$ we can use the same transformation $T$ to transform $F(\delta \phi, \delta b)=0$ into

$$
H(x, \delta b)=T^{T} F(T x, \delta b)=0 .
$$

The Jacobian $\frac{\partial}{\partial x} H(x, \delta b)=-T^{T} L\left(w\left(\phi^{*}\right)\right) T$ remains the same, and

$$
\frac{\partial}{\partial b} H(x, \delta b)=-T^{T} B \operatorname{diag}\left[v \circ \sin \left(B^{T} \phi\right)\right] .
$$

Therefore, since $\frac{\partial}{\partial x} H(x, \delta b)$ is nonsingular, we can apply again implicit function theorem to get

$$
\frac{d x(\delta b)}{d(\delta b)}=-\left(\frac{\partial}{\partial x} H(x, \delta b)\right)^{-1} \frac{\partial}{\partial b} H(x, \delta b) .
$$

Equation (11) follows after reverting the change of variables.

Now substituting (8), (10) and (11) into (9) we obtain

$$
\begin{aligned}
\frac{d w}{d b} & =\operatorname{diag}\left[v \circ \cos \left(B^{T} \phi^{*}\right)\right] \\
& +\operatorname{diag}\left[f(b) \circ \sin \left(B^{T} \phi\right)\right](R) \operatorname{diag}\left[v \circ \sin \left(B^{T} \phi^{*}\right)\right]
\end{aligned}
$$

where $R=B^{T} L(w)^{\dagger} B$ is the effective resistance matrix when the weights $w$ are interpreted as conductances. $R_{i j, k l}$ represents the voltage difference between nodes $i$ and $j$ when a current of 1 unit is injected in $k$ and subtracted from $l$ [7]. Notice that in our case, it is possible that some of the weights $w_{i j}$ are negative. Nonetheless $L(w)$ is positive semi-definite with a single zero eigenvalue and therefore $R_{i j, i j}>0$. Thus, we can still interpret $R_{i j, i j}$ as a measure of the distance between $i$ and $j$.

Clearly, using this notion of distance, one can see how $\nu_{2}(w)$ is more sensitive to changes between nodes "farther" away. However, what is interesting here is the appearance of the term $\cos \left(\phi_{j}^{*}-\phi_{i}^{*}\right)$. When the phase difference between certain buses is larger than $\frac{\pi}{2}$ we have $\cos \left(\phi_{j}^{*}-\phi_{i}^{*}\right)<0$ and therefore and increment on $b_{i j}$ could possibly affect negatively the weight $w_{i j}(\phi)$. This phenomenon is numerically illustrated in Section V-B.

\section{NUMERICAL EXAMPLES}

In this section, two examples are provided to illustrate the findings of the previous sections.

\section{A. Generator Power Scheduling}

Consider a network of 3 generator buses, and 3 load buses disposed in a complete graph configuration as in Figure 2 with $b_{i i}=10$ for every generator, $b_{12}=b_{13}=2$ and $b_{23}=$ 10 . The initial power scheduling is

$$
P_{d}=\left[\begin{array}{lll}
4 & 6 & 8
\end{array}\right]^{T} \text {, and } P_{g}=\left[\begin{array}{lll}
7.994 & 3.006 & 7
\end{array}\right]^{T} \text {. }
$$

Assume also that the power demanded in each bus is fixed and cannot be modified by the algorithm.

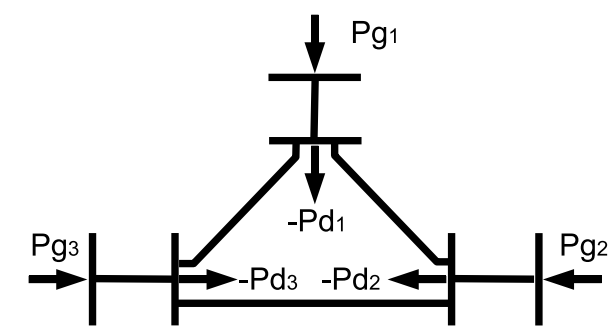

Fig. 2. 3 Bus Power Network

We now see how the operating point of the network can be locally improved. One possible equilibrium $\phi^{*}$ that solves (6), for given $P$, is

$$
\phi^{*}=\left[\begin{array}{llllll}
.513 & 0 & .032 & .808 & .097 & .279
\end{array}\right]^{T} \pi .
$$

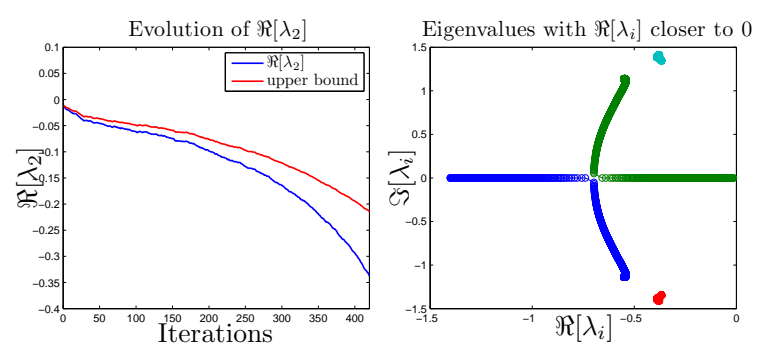

Fig. 3. Evolution of $\Re\left[\lambda_{2}\right]$

The values of $P$ chosen are such that the system is very close to the bifurcation. Figure 3 shows the evolution of $\Re\left[\lambda_{2}\right]$, the corresponding upper bound provided in Theorem 1 and the location of the 4 th closest eigenvalues to the imaginary axis. We can see that when the system is close to a bifurcation, not only our proposed adaptation is more effective, but also the upper bound computed is tighter.

Initially $\Re\left[\lambda_{2}\right]=\lambda_{2}$ is very close to zero, but it gradually decreases until a new eigenvalue with non zero imaginary part becomes dominant. This is captured in the right graph of Figure 3. After this point, $\Re\left[\lambda_{2}\right]=-\frac{d\left(x_{i}\right)}{2 m\left(x_{i}\right)}$ and the dependence on the algebraic connectivity is lost.

\section{B. Adding or Removing a Line}

In this example each generator bus $g_{i}$ is generating $P_{g_{i}}=$ $\bar{P}$ and each load bus demands a power of $P_{d_{i}}=\bar{P}$ with $\bar{P}=5$. We assume $b_{i j}=10 \forall i j$.

Among the possible equilibria for this network we study the equilibrium $\phi_{2}^{*}$ given by $\left(\phi_{2}^{*}\right)_{d_{i}}=\frac{2 \pi}{6}(i-1), i \in$ $\{1, \ldots, 6\}$ and $\left(\phi_{2}^{*}\right)_{g_{i}}=\left(\phi_{2}^{*}\right)_{d_{i}}+\arcsin \left(\frac{1}{2}\right)^{6}$. 


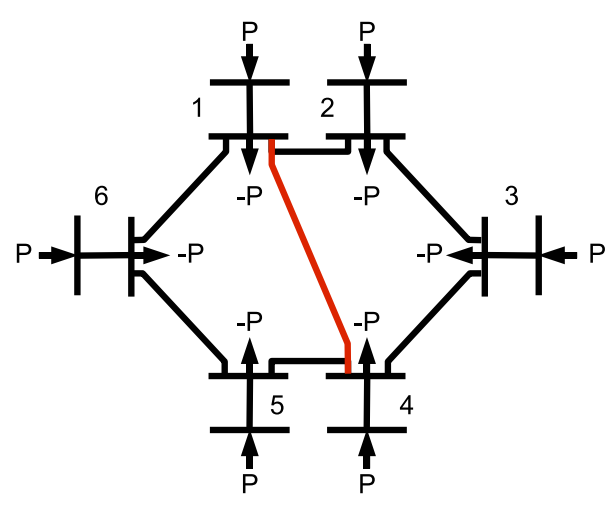

Fig. 4. 6 Bus Power Network

In this case, when we add a line between $d_{1}$ and $d_{4}$, i.e. we increase $\sigma b_{d_{1} d_{4}}$ (in red) from $\sigma=0$ to $\sigma=1, \frac{d w}{d b}$ becomes

$$
\frac{d w}{d b_{d_{1} d_{4}}}=-e_{d_{1} d_{4}}<0,
$$

since $\phi_{d_{4}}^{*}-\phi_{d_{1}}^{*}=\pi$, and therefore $\left\langle\nabla \nu_{2}, \delta w\right\rangle \leq 0$. In fact, since $x^{*}\left(w\left(\phi_{2}^{*}\right)\right)_{d 1} \neq x^{*}\left(w\left(\phi_{2}^{*}\right)\right)_{d 4}$, we can see in Figure 5 that $\nu_{2}\left(w\left(\phi_{2}^{*}\right)\right)$ decreases.
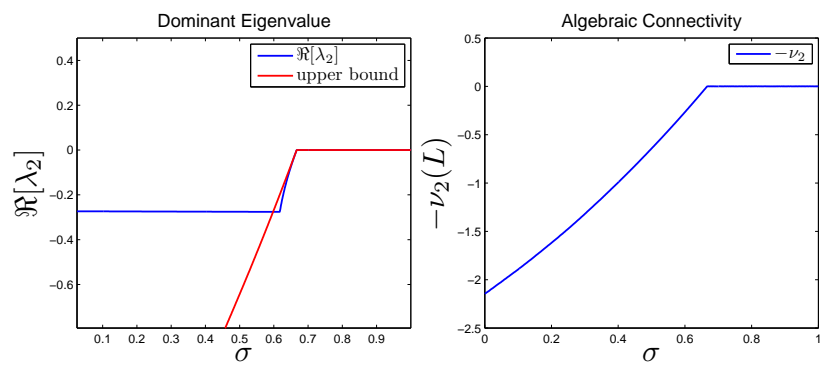

Fig. 5. Effect of Adding $b_{d_{1} d_{4}}$

Here we can also see that although $\Re\left[\lambda_{2}\right]$ and $\nu_{2}\left(L\left(w\left(\phi^{*}\right)\right)\right)$ might not be in general correlated, when the system is close to a bifurcation, i.e. $\nu_{2}\left(L\left(w\left(\phi^{*}\right)\right)\right)$ and $\Re\left[\lambda_{2}\right]$ are close to zero, the changes on $\nu_{2}\left(L\left(w\left(\phi^{*}\right)\right)\right)$ directly affect $\Re\left[\lambda_{2}\right]$. Moreover, Figure 5 shows how our upper-bound is only valid in this specific case.

\section{CONCLUSion ANd Future WORK}

This paper studies the effect of power scheduling and line impedances on the damping of a power network. Using implicit function theorem, we derive the dependence of the operating point on network parameters. This allows us to design several updating directions for the network parameters that locally increase the damping and discover some interesting behaviors, for instance, adding a line can degrade the damping and removing a line can improve it.

There are several directions along which this work can be extended. For example, recent developments in the context of coupled oscillators [13] have related the instability of equilibria with specific partitions of the network. It would be interesting and useful to see to what extent those results are related and can be applied to the subject of this paper. Additionally, there has been increasing interest in trying to include stability in the computation of the Optimal Power Flow. This is usually implemented by adding extra constraints to the minimization problem [6]. The results presented here unveil the tradeoff between stability and cost efficiency, and can potentially be useful to include stability as part of the optimization objective.

Acknowledgments: The research is supported by NSF under CCF-0835706.

\section{REFERENCES}

[1] A.R. Bergen and D.J. Hill. A structure preserving model for power system stability analysis. IEEE Transactions on Power Apparatus and Systems, PAS-100(1):25-35, Jan. 1981.

[2] Stephen Boyd. Convex optimization of graph laplacian eigenvalues. In International Congress of Mathematicians, pages 1311-1319.

[3] Chia-Chi Chu. Transient dynamics of electric power systems : direct stability assessment and chaotic motions. $\mathrm{PhD}$ thesis, Electrical and Computer Engineer, Cornell University, 1996.

[4] I. Dobson. Observations on the geometry of saddle node bifurcation and voltage collapse in electrical power systems. Circuits and Systems I: Fundamental Theory and Applications, IEEE Transactions on, 39(3):240 -243, mar. 1992.

[5] F. Dorfler and F. Bullo. Transient stability analysis in power networks and synchronization of non-uniform Kuramoto oscillators. pages 930937, Baltimore, MD, June 2010.

[6] D. Gan, R.J. Thomas, and R.D. Zimmerman. Stability-constrained optimal power flow. Power Systems, IEEE Transactions on, 15(2):535 -540 , may. 2000.

[7] Arpita Ghosh, Stephen Boyd, and Amin Saberi. Minimizing effective resistance of a graph. SIAM Rev., 50(1):37-66, 2008.

[8] I. Gohberg, P. Lancaster, and L. Rodman. Matrix Polynomials. Academic Press, 1982.

[9] D.J. Gotham and G.T. Heydt. Power flow control and power flow studies for systems with facts devices. Power Systems, IEEE Transactions on, 13(1):60 -65, feb. 1998.

[10] J.-B. Hiriart-Urruty and A.S. Lewis. The clarke and michelpenot subdifferentials of the eigenvalues of a symmetric matrix. Computational Optimization and Applications, 13:13-23, 1999. 10.1023/A:1008644520093.

[11] Sung-Kwan Joo, Jang-Chul Kim, and Chen-Ching Liu. Empirical analysis of the impact of 2003 blackout on security values of u.s. utilities and electrical equipment manufacturing firms. Power Systems, IEEE Transactions on, 22(3):1012 -1018, aug. 2007.

[12] John M. Lee. Intorduction to Smooth Manifolds. Springer, 2003.

[13] E. Mallada and A. Tang. Synchronization of phase-coupled oscillators with arbitrary topology. In Proceedings of American Control Conference, 2010.

[14] A. Mas-Colell, M.D. Whinston, and J.R. Green. Microeconomic theory. Oxford University Press New York, 1995.

[15] Hadi Saadat. Power System Analysis. McGraw Hill, 2nd edition edition, 2002.

[16] Francoise Tisseur and Karl Meerbergen. The quadratic eigenvalue problem. SIAM Review, 43(2):235-286, 2001. 